Check for updates

Cite this: RSC Adv., 2018, 8, 40446

Received 19th August 2018

Accepted 17th November 2018

DOI: $10.1039 / \mathrm{c} 8 \mathrm{ra06} 638 \mathrm{~g}$

rsc.li/rsc-advances

\section{A straightforward and convenient synthesis of functionalized allyl thiosulfonates and allyl disulfanes $\uparrow$}

\author{
Raju Jannapu Reddy, (D)* Md. Waheed $\ddagger$ and Jangam Jagadesh Kumarł
}

A practical, highly flexible and eco-friendly method has been developed for the synthesis of allyl thiosulfonates using Morita-Baylis-Hillman (MBH) allyl bromides and sodium arylthiosulfonates, which were readily assembled without any reagent/catalyst. Moreover, the allyl thiosulfonates were successfully transformed into a set of two synthetically viable diallyl disulfanes and unsymmetrical allyl disulfanes in the presence of $\mathrm{Cs}_{2} \mathrm{CO}_{3}$. The present protocols are operationally simple and convenient to generate a wide range of functionalized allyl thiosulfonates and allyl disulfanes in good to excellent yields.

\section{Introduction}

Organosulfur compounds have received much attention in recent years. ${ }^{1}$ The direct construction of a carbon-sulfur bond is a potent method in modern organic synthesis. ${ }^{2}$ Among a variety of organosulfur compounds, the thiosulfonates (RS$\left.\mathrm{SO}_{2} \mathrm{R}^{1}\right)^{3}$ and the disulfanes (RS-SR ${ }^{4}$ are a privileged class of compounds and they serve as electrophilic sulfenylating agents in organic synthesis. Additionally, the thiosulfonates and the disulfanes have several advantages, which include being an odourless sulfur source, higher reactivity, and better stability than other frequently used sulfenylating agents. In general, the thiosulfonates have shown a broad spectrum of clinical properties like antiviral, antimicrobial and fungicidal activities. $^{5}$ A wide range of effective strategies have been established for the synthesis of $S$-aryl arenethiosulfonates. ${ }^{6-8}$ Although the reactions of sodium thiosulfonates with alkyl halides have been studied, ${ }^{9}$ a general method is still highly desirable. This fact motivated us to develop a new strategy for the synthesis of functionalized allyl thiosulfonates.

In contrast, the disulfanes also have significant importance in pharmaceutical chemistry ${ }^{\mathbf{1 0}}$ and chemical biology. ${ }^{\mathbf{1 1}}$ The S-S bond plays an indispensable role in allium compound $\mathbf{s}^{\mathbf{1 0 a}}$ and disulfiram, ${ }^{10 \boldsymbol{b}-\boldsymbol{d}}$ which act as anti-thrombotic and antialcoholic drugs (Scheme 1). Numerous synthetic methods have been reported, which rely on the oxidative coupling of thiols to construct the S-S bond. ${ }^{12}$ However, these methods have some disadvantages, either they involve the tedious use

Department of Chemistry, Osmania University, Hyderabad 500 007, India. E-mail: rajuchem77@osmania.ac.in; rajuchemo8@yahoo.co.in

$\dagger$ Electronic supplementary information (ESI) available: General experimental procedures, spectroscopic data and NMR spectra for the new compounds. See DOI: $10.1039 / \mathrm{c} 8 \mathrm{ra06938g}$

\$ These authors contributed equally. of thiols or their equivalents or they are limited by commercial scarcity. Therefore, it is still necessary to develop a facile methodology for the synthesis of allyl disulfides from easily accessible starting materials.

According to retrosynthetic analysis, the proposed strategy has a great advantage of extensively available starting materials (Scheme 1). In fact, the allyl bromides can be derived from Morita-Baylis-Hillman (MBH) adducts $^{\mathbf{1 3}}$ and sodium thiosulfonates easily prepared from sulfinate salts. ${ }^{\mathbf{1 4}}$ In particular, the sodium thiosulfonate precursors are preferable to use as sulfenylating agents since they are bench stable and generally crystalline solids with low toxicity. ${ }^{\mathbf{1 4 b}}$ We have also envisaged that the allyl thiosulfonates would be converted into the corresponding unprecedented allyl disulfanes. In a continuation of our interest in organosulfur chemistry, ${ }^{15}$ we decided to study $\mathrm{MBH}$ bromide with sodium thiosulfonate for direct $\mathrm{C}-\mathrm{S}$ bond construction, which represents an interesting and challenging endeavour in organic synthesis. In this context, we disclose our findings and report the scope for the

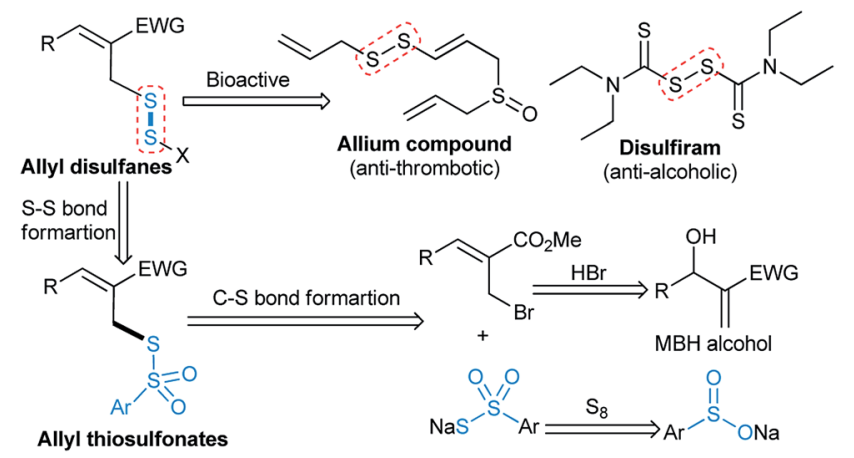

Scheme 1 Strategy for synthesis of allyl thiosulfonates and allyl disulfanes. 
synthesis of unsymmetrical allyl thiosulfonates and allyl disulfanes with a variety of substitution patterns.

\section{Results and discussion}

At the outset, optimization studies were undertaken (see ESI $\dagger$ ) for the nucleophilic substitution reaction of (Z)-methyl 2(bromo-methyl)-3-phenylacrylate (1a) with sodium thiosulfonate (2a) as a model substrate. To our delight, an excellent yield (94\%) of the desired allyl thiosulfonate (3aa) was achieved with 1.5 equiv. of $2 \mathrm{a}$ in $\mathrm{CH}_{3} \mathrm{CN}$ at room temperature within $2 \mathrm{~h}$. Then we explored a broad range of allyl bromides $(\mathbf{1 a}-\mathbf{t})$ with sodium thiosulfonates (2a-d) for the synthesis of allyl thiosulfonates 3aa-tb (Table 1). In addition to 3aa, the 4-bromo and 4-chlorosubstituted allyl bromides (1) and $\mathbf{1 c})$ readily reacted with $\mathbf{2 a}$, giving the desired thiosulfonates (3ba-ca) in $89 \%$ and $84 \%$ yields, respectively. Various aryl-substituted MBH bromides (1a-h) smoothly reacted with sodium 4-methylbenzenesulfonothioate $\mathbf{2 b}$ to provide the desired products ( $3 \mathbf{a b}-$ hb) in $70-92 \%$ yield. The 2-naphthyl derived thiosulfonates (3ia and $3 \mathbf{j b} \mathbf{b}$ ) were obtained in high yield under optimised reaction conditions. Heteroaryl derived allyl bromide (1k) also served as a good substrate, affording $3 \mathbf{k b}$ in $68 \%$ yield. Moreover,

Table 1 Substrate scope for synthesis of allyl thiosulfonates ${ }^{a, b}$
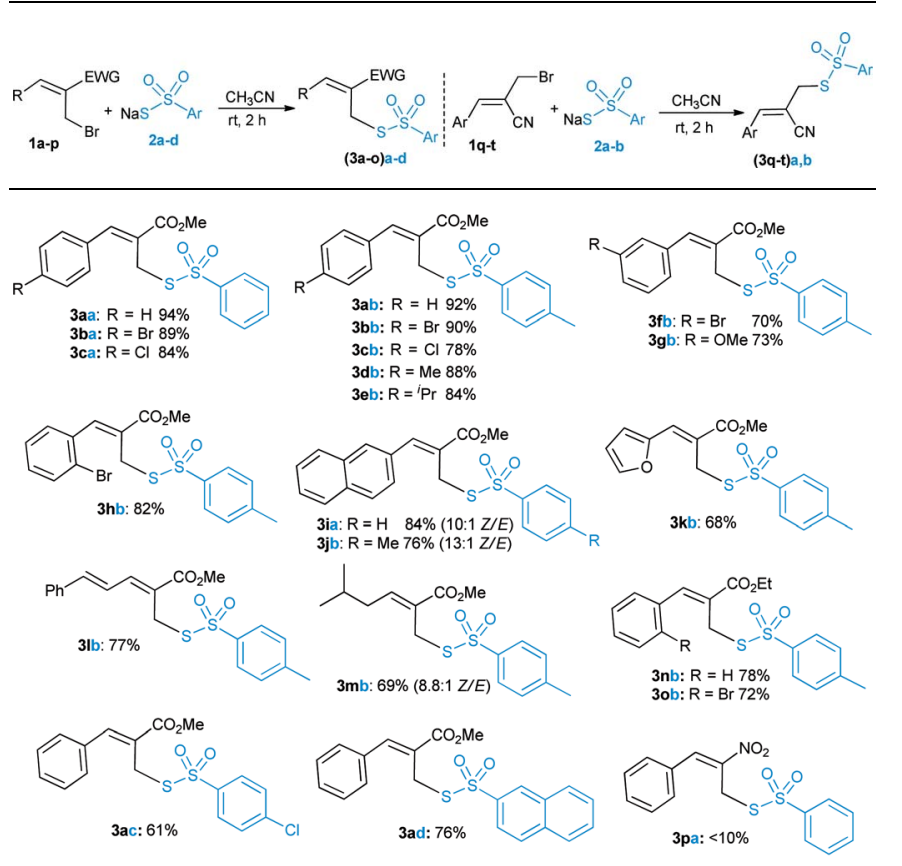

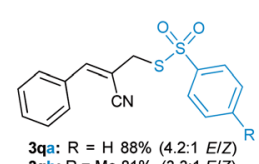

3qa: $R=H 88 \%(4.2: 1 \mathrm{EIZ})$
3qb: $R=\operatorname{Me} 81 \%(3.3: 1 \mathrm{EIZ})$

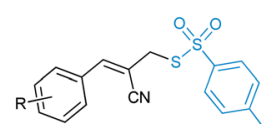

3rb: $R=4^{-} \operatorname{Pr} 78 \% \quad(5.0: 1 \mathrm{E} / Z)$ 3sb: $\mathrm{R}=3-\mathrm{OMe} 71 \%(\mathbf{4} .6: 1 \mathrm{EIZ})$

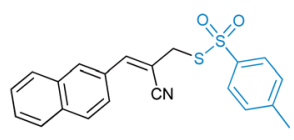

3tb: $84 \%(2.7: 1 E I Z)$
${ }^{a}$ All reactions performed on $0.4 \mathrm{mmol}$ of $1 \mathrm{a}-\mathrm{t}$ (1 eq.), sodium thiosulfonates $2 \mathrm{a}-\mathbf{d}$ (1.5 eq.) in $\mathrm{CH}_{3} \mathrm{CN}(2 \mathrm{~mL})$ at room temperature. ${ }^{b}$ Isolated yields. Reactions performed on a $4 \mathrm{mmol}$ scale for synthesis of $3 \mathbf{a a} / \mathbf{b}$ and $2 \mathrm{mmol}$ scale for synthesis of $3 \mathbf{b a},(3 \mathbf{b}-\mathbf{e}, \mathbf{g}-\mathbf{i}, \mathbf{k}, \mathbf{n}, \mathbf{o}, \mathbf{q})$ b and 3ad (see ESI).

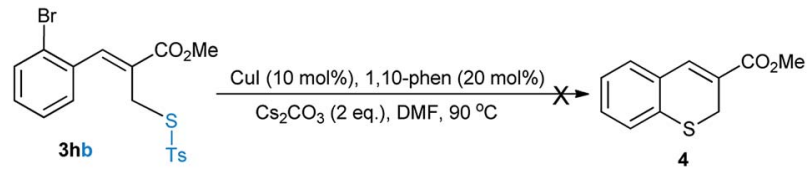

Scheme 2 Synthesis of methyl $2 \mathrm{H}$-thiochromene-3-carboxylate (4).

cinnamyl and isovaleraldehyde derived allyl bromides (11-m) were also suitable substrates to produce the desired allyl thiosulfonates (3lb and $\mathbf{3} \mathbf{m b}$ ) in high yields. The ethyl ester derived MBH bromides (1n and 1o) provided the corresponding products $3 \mathbf{n b}$ and $3 \mathbf{o b}$ in $78 \%$ and $72 \%$ yields, respectively. Disappointingly, the nitrostyrene derived bromide (1p) was sluggish to react with 2a. The substrate scope was further extended to acrylonitrile derived MBH bromides (1q-t). This successfully achieved the corresponding thiosulfonates $(3 \mathbf{q a} / \mathbf{b}, \mathbf{3}(\mathbf{r}-\mathbf{t}) \mathbf{b})$ in moderate to good yields with inseparable regioisomers (ESI $\dagger$ ). ${ }^{16}$ Overall, these reactions were scalable to provide the desired allyl thiosulfonates with a little deviation in the outcome (ESI†).

Next, we explored the synthetic utility of readily accessible allyl thiosulfonates in organic synthesis. We desired to synthesise methyl $2 H$-thiochromene-3-carboxylate $(4)^{17}$ via the cyclization of 2-bromo derived thiosulfonate $3 \mathbf{h b}$ (Scheme 2). Unfortunately, we did not attain the anticipated cyclic product 4; however, other interesting allyl disulfanes were obtained under these conditions (Table 2). Hence, we directed our attention toward the synthesis of allyl disulfanes using allyl thiosulfonates.

Encouraged by this result, we commenced the optimization of reaction conditions using thiosulfonates $\mathbf{3} \mathbf{a b}$ (Table 2). The use of $\mathrm{CuI}(10 \mathrm{~mol} \%)$ and $\mathrm{CuBr}(10 \mathrm{~mol} \%)$ with 1,10-phen (20 mol\%) in the presence of $\mathrm{Cs}_{2} \mathrm{CO}_{3}$ (2 eq.) in DMF afforded the desired disulfanes (5a and $\mathbf{6 a}$ ) in low yield (entries 1 and 2). There was no reaction in the presence of $\mathrm{K}_{2} \mathrm{CO}_{3}$ in DMF; however, substantial progress was seen in toluene and THF (entries 3-5). In the absence of a ligand (1,10-phen), the reaction proceeded with $5 \mathrm{~mol} \%$ of $\mathrm{CuBr}$, but there was no reaction with only $\mathrm{CuBr}$ and without $\mathrm{Cs}_{2} \mathrm{CO}_{3}$ (entries 6-8). To our surprise, $\mathrm{Cs}_{2} \mathrm{CO}_{3}$ alone afforded the desired disulfanes with improved yields (entries 9-11). A satisfactory yield of diallyl disulfane 5a $(49 \%)$ and unsymmetrical disulfane $\mathbf{6 a}(44 \%)$ were obtained when the reaction was carried out using 1.0 eq. of $\mathrm{Cs}_{2} \mathrm{CO}_{3}$ in THF at $60{ }^{\circ} \mathrm{C}$ (entry 10). Low yields were obtained with DBU, DABCO, and $\mathrm{K}_{2} \mathrm{CO}_{3}$ (entries 12-14) and no reaction was found without a base (entry 15). It is worth noting that our attempts to produce as a single product either $\mathbf{5 a}$ or $\mathbf{6 a}$ were unsuccessful.

With the optimized conditions in hand, the scope of the reaction was further verified and the results are summarized in Table 3. Pleasingly, the allyl thiosulfonate substrates (3a, d, e, $\mathbf{g}$, $\mathbf{h}, \mathbf{o} / \mathbf{b}$ and $\mathbf{3 a a} / \mathbf{b}$ ) bearing different groups at the para, meta or ortho position of the aromatic rings proceeded efficiently to afford the corresponding allyl disulfanes (5a-g and $\mathbf{6 a}-\mathbf{h})$ in good to high yields. The bulky 2-naphthalene derived thiosulfonates $(3 \mathbf{j b} \mathbf{b} / \mathbf{3 a d})$ were also well tolerated to provide the diallyl disulfanes $(5 \mathbf{h} / \mathbf{a})$ and unsymmetrical disulfanes $(6 \mathbf{6} / \mathbf{j})$ in good yields. The ethyl ester derived thiosulfonate (3nb) was also a good substrate to furnish allyl disulfane $5 \mathbf{i}$ and $\mathbf{6 k}$ in $40 \%$ and 
Table 2 Optimization for synthesis of allyl disulfanes from thiosulfonate $3 a b^{a}$

\begin{tabular}{|c|c|c|c|c|}
\hline Entry & Reaction conditions & Time & $5 \mathbf{a}^{b}$ & $6 \mathbf{a}^{b}$ \\
\hline 1 & $\mathrm{CuI}(10 \mathrm{~mol} \%), 1,10$-phen $(20 \mathrm{~mol} \%), \mathrm{Cs}_{2} \mathrm{CO}_{3}(2 \mathrm{eq}),. \mathrm{DMF}, 90^{\circ} \mathrm{C}$ & $6 \mathrm{~h}$ & $20 \%$ & $14 \%$ \\
\hline 2 & $\mathrm{CuBr}(10 \mathrm{~mol} \%), 1,10$-phen $(20 \mathrm{~mol} \%), \mathrm{Cs}_{2} \mathrm{CO}_{3}$ (2 eq.), DMF, $90^{\circ} \mathrm{C}$ & $6 \mathrm{~h}$ & $29 \%$ & $20 \%$ \\
\hline 3 & $\mathrm{CuBr}(10 \mathrm{~mol} \%), 1,10$-phen $(20 \mathrm{~mol} \%), \mathrm{K}_{2} \mathrm{CO}_{3}$ (2 eq.), DMF, $90^{\circ} \mathrm{C}$ & $6 \mathrm{~h}$ & NR & NR \\
\hline 4 & $\mathrm{CuBr}(10 \mathrm{~mol} \%), 1,10$-phen $(20 \mathrm{~mol} \%), \mathrm{Cs}_{2} \mathrm{CO}_{3}$ (2 eq.), toluene, $90{ }^{\circ} \mathrm{C}$ & $6 \mathrm{~h}$ & $34 \%$ & $11 \%$ \\
\hline 5 & $\mathrm{CuBr}(10 \mathrm{~mol} \%), 1,10$-phen (20 mol\%), $\mathrm{Cs}_{2} \mathrm{CO}_{3}$ (2 eq.), THF, $60{ }^{\circ} \mathrm{C}$ & $4 \mathrm{~h}$ & $30 \%$ & $20 \%$ \\
\hline 6 & $\mathrm{CuBr}(10 \mathrm{~mol} \%), \mathrm{Cs}_{2} \mathrm{CO}_{3}$ (2 eq.), THF, $60^{\circ} \mathrm{C}$ & $4 \mathrm{~h}$ & $35 \%$ & $22 \%$ \\
\hline 7 & $\mathrm{CuBr}(5 \mathrm{~mol} \%), \mathrm{Cs}_{2} \mathrm{CO}_{3}$ (2 eq.), THF, $60^{\circ} \mathrm{C}$ & $4 \mathrm{~h}$ & $38 \%$ & $24 \%$ \\
\hline 8 & $\mathrm{CuBr}(10 \mathrm{~mol} \%)$, THF, $60{ }^{\circ} \mathrm{C}$ & $6 \mathrm{~h}$ & NR & NR \\
\hline 9 & $\mathrm{Cs}_{2} \mathrm{CO}_{3}$ (2 eq.), THF, $60^{\circ} \mathrm{C}$ & $2 \mathrm{~h}$ & $45 \%$ & $36 \%$ \\
\hline 10 & $\mathrm{Cs}_{2} \mathrm{CO}_{3}$ (1 eq.), THF, $60^{\circ} \mathrm{C}$ & $2 \mathrm{~h}$ & $49 \%$ & $44 \%$ \\
\hline 11 & $\mathrm{Cs}_{2} \mathrm{CO}_{3}$ (0.5 eq.), THF, $60^{\circ} \mathrm{C}$ & $4 \mathrm{~h}$ & $38 \%$ & $29 \%$ \\
\hline 12 & DBU ( 2 eq.), THF, $60^{\circ} \mathrm{C}$ & $6 \mathrm{~h}$ & $20 \%$ & $20 \%$ \\
\hline 13 & $\operatorname{DABCO}(2$ eq. $), \mathrm{THF}, 60^{\circ} \mathrm{C}$ & $6 \mathrm{~h}$ & NR & NR \\
\hline 14 & $\mathrm{~K}_{2} \mathrm{CO}_{3}$ (2 eq.), THF, $60^{\circ} \mathrm{C}$ & $6 \mathrm{~h}$ & NR & NR \\
\hline 15 & Without base, THF, $60^{\circ} \mathrm{C}$ & $6 \mathrm{~h}$ & NR & NR \\
\hline
\end{tabular}

${ }^{a}$ All reactions performed on a $0.4 \mathrm{mmol}$ scale of $3 \mathbf{a b}$ in solvent $(2 \mathrm{~mL}) .{ }^{b}$ Isolated yields. NR: no reaction.

$35 \%$ yields, respectively. But an extension to other thiosulfonates $\mathbf{3 k b}$ and $\mathbf{3 q b}$ failed to provide the desired allyl disulfides.

Recently, Jiang and co-workers ${ }^{\mathbf{1 8}}$ demonstrated the potential application of $S$-acetyl disulfanes in oxidative coupling with arylboronic acids. In this direction, the allyl thiosulfonates 3(a/ h)b were treated with potassium thioacetate in DMF and the anticipated products $(7 \mathbf{a} / \mathbf{b})$ were achieved in $88 \%$ and $85 \%$ yields, respectively (Scheme 3). Sequential one-pot nucleophilic substitution reactions were performed using $\mathrm{MBH}$ bromide 1a with sodium thiosulfonate (2b) followed by potassium thioacetate (Scheme 3). The desired product 7a was obtained in 78\% yield and this procedure has notable advantages, such as straightforwardness, step-economy and scalability in a one-pot operation (see ESI†).

To gain more insight, the $S$-aryl arenethiosulfonates $(\mathbf{8 a}$ and 8b) were treated with $\mathrm{Cs}_{2} \mathrm{CO}_{3}$ under the same reaction conditions. Thus, it was found that no reaction representing the allyl moiety played a vital role in the formation of disulfanes. A radical-trapping experiment was also performed with 3ab using TEMPO (2 equiv.) as a radical scavenger under standard conditions. The disulfanes $5 \mathbf{a}$ and $\mathbf{6 a}$ were isolated in low yields, alongside the TEMPO derived product (9), ${ }^{4 \boldsymbol{d}}$ as detected by HRMS (Scheme 4b). These experimental results indicate that the reaction may proceed via a radical pathway leading to the formation of allyl disulfanes $\mathbf{5}$ and $\mathbf{6}$.

\section{Experimental}

General procedure-1 (GP1) for synthesis of allyl thiosulfonates

A heat gun-dried Schlenk tube was charged with Morita-BaylisHillman allyl bromides 1a-t ( $0.4 \mathrm{mmol}, 1.0$ equiv.) and sodium thiosulfonates $2 \mathrm{a}-\mathbf{d}$ (0.6 mmol, 1.5 equiv.) in $\mathrm{CH}_{3} \mathrm{CN}(2.0 \mathrm{~mL})$. The reaction mixture was stirred at room temperature for $2 \mathrm{~h}$ and monitored by TLC until it was either complete or appeared to be making no further progress. The mixture was quenched by the addition of water $(10 \mathrm{~mL})$ followed by extraction with EtOAc $(3 \times 10 \mathrm{~mL})$. The combined organic layers were washed with brine $(2 \times 10 \mathrm{~mL})$, dried over anhydrous $\mathrm{Na}_{2} \mathrm{SO}_{4}$, and the solvent was removed under reduced pressure. The resulting residue was subjected to flash chromatography (silica gel, eluted with $20-30 \%$ ethyl acetate/petether) to afford the desired allyl thiosulfonates.

\section{Allyl thiosulfonate 3aa}

Prepared according to GP1 using $1 \mathrm{a}(101.2 \mathrm{mg}, 0.4 \mathrm{mmol})$ with sodium benzenethiosulfonate $2 \mathrm{a}(117.6 \mathrm{mg}, 0.6 \mathrm{mmol})$ to afford 3aa (130.6 mg, 94\%) using 20\% ethyl acetate/petether as a colorless solid. Mp: 95-97 ${ }^{\circ} \mathrm{C} ;{ }^{1} \mathrm{H}$ NMR (400 MHz, $\mathrm{CDCl}_{3}$ ) $\delta$ 7.87-7.77 (m, 3H), $7.63(\mathrm{t}, J=7.4 \mathrm{~Hz}, 1 \mathrm{H}), 7.51(\mathrm{t}, J=7.8 \mathrm{~Hz}$, 2H), 7.42-7.32 (m, 5H), $4.17(\mathrm{~s}, 2 \mathrm{H}), 3.75(\mathrm{~s}, 3 \mathrm{H}) ;{ }^{13} \mathrm{C}$ NMR (101 $\left.\mathrm{MHz} \mathrm{CDCl}_{3}\right) \delta$ 166.8, 144.6, 144.3, 134.0, 133.7, 129.8, 129.7 (2C), 129.4 (2C), 129.0 (2C), 127.2 (2C), 124.4, 52.6, 33.4; HRMS (ESI) calculated for $\mathrm{C}_{17} \mathrm{H}_{16} \mathrm{O}_{4} \mathrm{~S}_{2} \mathrm{Na}[\mathrm{M}+\mathrm{Na}]^{+}: \mathrm{m} / z$ 371.0388, found 371.0389. Prepared according to GP1 using 1a (1.02 g, 4.0 mmol) with sodium benzenethiosulfonate $2 \mathrm{a}(1.47 \mathrm{~g}, 6.0 \mathrm{mmol})$ in $\mathrm{CH}_{3} \mathrm{CN}(20 \mathrm{~mL})$ to afford $3 \mathrm{aa}(1.1 \mathrm{~g}, 79 \%)$ as a colorless solid.

\section{General procedure-2 (GP2) for synthesis of allyl disulfanes}

A heat gun-dried Schlenk tube was charged with allyl thiosulfonates 3 (0.8 mmol, 1.0 equiv.) and cesium carbonate (260.6 mg, $0.8 \mathrm{mmol}, 1.0$ equiv.) in dry THF (4.0 mL). The reaction mixture was stirred at $60{ }^{\circ} \mathrm{C}$ for $2 \mathrm{~h}$ and monitored by TLC either until it was complete or appeared to be making no further progress. The mixture was allowed to cool to room temperature and quenched by the addition of water $(20 \mathrm{~mL})$ 
Table 3 Substrate scope for synthesis of allyl disulfanes ${ }^{a, b}$
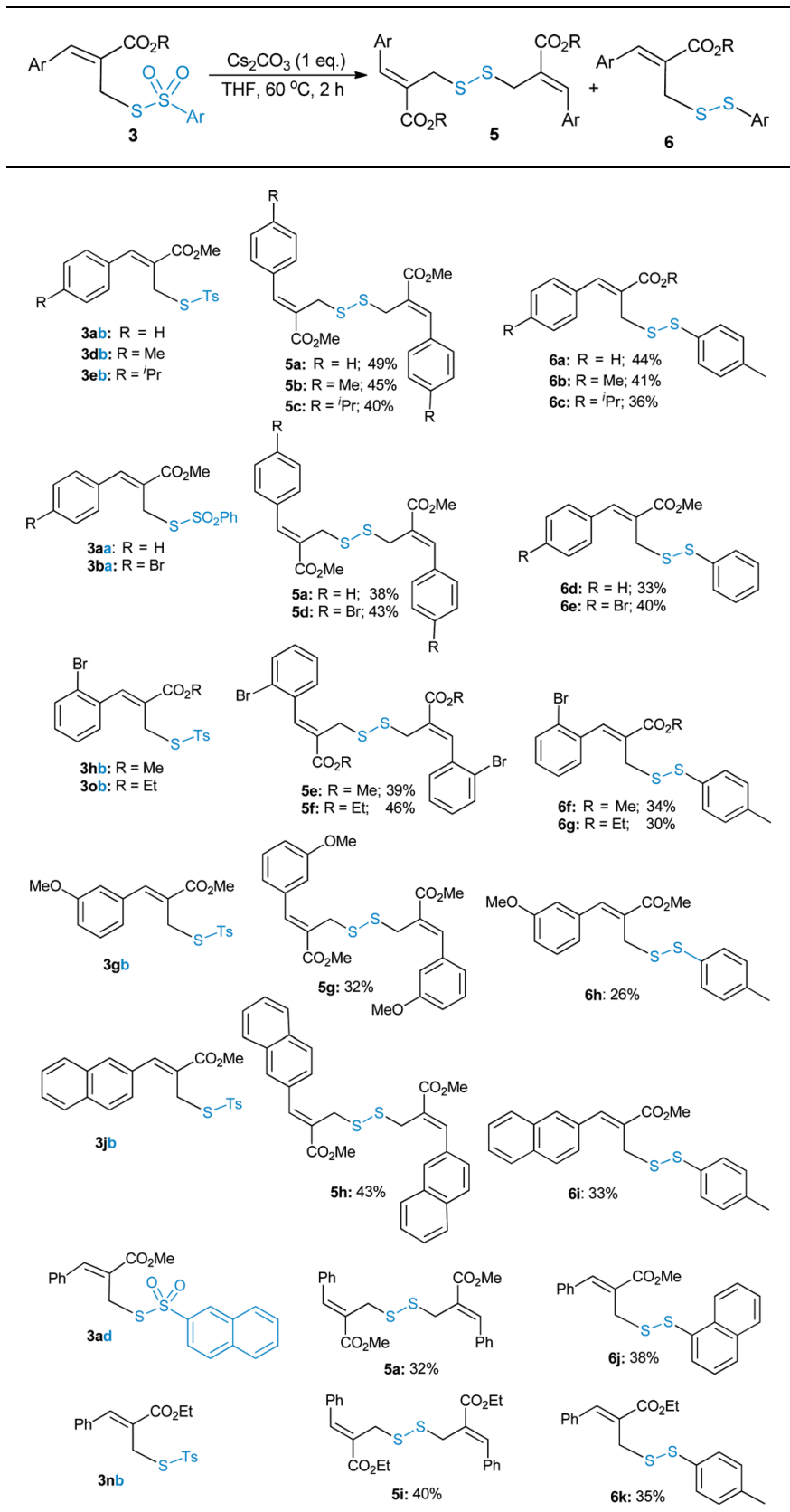

${ }^{a}$ All reactions performed on $0.8 \mathrm{mmol}$ of allyl thiosulfonates (1 eq.) and $\mathrm{Cs}_{2} \mathrm{CO}_{3}$ (1.0 eq.) in dry THF $(4 \mathrm{~mL})$ at $60{ }^{\circ} \mathrm{C}$ for $2 \mathrm{~h} .{ }^{b}$ Isolated yields.

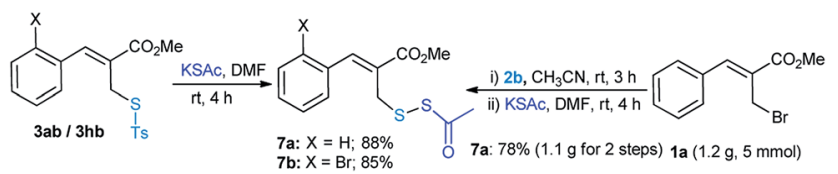

Scheme 3 Reactions performed on $0.8 \mathrm{mmol}$ of $3 \mathrm{ab} / 3 \mathrm{hb}$ with KSAc (1.5 eq.). A gram scale reaction of allyl bromide $1 \mathrm{a}$ with $2 \mathrm{~b} / \mathrm{KSAc}$ for the synthesis of $7 a$.

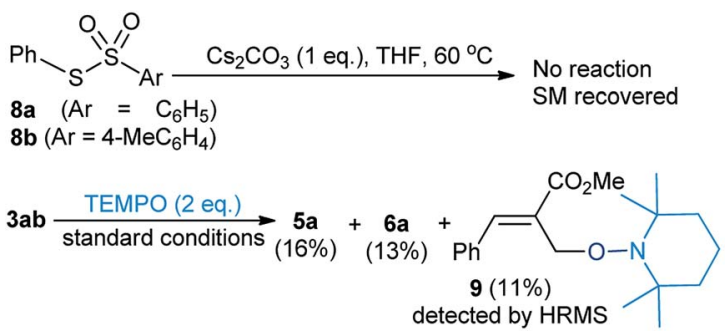

(a)

(b)

Scheme 4 Control experiments.

followed by extraction with EtOAc $(3 \times 30 \mathrm{~mL})$. The combined organic layers were washed with brine $(2 \times 20 \mathrm{~mL})$, dried over anhydrous $\mathrm{Na}_{2} \mathrm{SO}_{4}$, and the solvent was removed under reduced pressure. The resulting residue was subjected to flash chromatography (silica gel, eluted with $20-30 \%$ ethyl acetate/ petether) to afford desired allyl disulfanes $\mathbf{5}$ and $\mathbf{6}$.

\section{Diallyl disulfane 5a}

Prepared according to GP2 using 3ab (289.6 mg, $0.8 \mathrm{mmol})$ with $\mathrm{Cs}_{2} \mathrm{CO}_{3}(260.6 \mathrm{mg}, 0.8 \mathrm{mmol})$ to afford $5 \mathbf{a}(81.2 \mathrm{mg}, 24.5 \% \times 2=$ $49 \%$ ) using $20 \%$ ethyl acetate/petether as a colorless solid. Mp: 110-112 ${ }^{\circ} \mathrm{C} ;{ }^{1} \mathrm{H}$ NMR $\left(400 \mathrm{MHz}, \mathrm{CDCl}_{3}\right) \delta 7.82(\mathrm{~s}, 1 \mathrm{H}), 7.51(\mathrm{dd}, J$ $=7.3,1.0 \mathrm{~Hz}, 2 \mathrm{H}), 7.41-7.34(\mathrm{~m}, 3 \mathrm{H}), 3.88(\mathrm{~s}, 2 \mathrm{H}), 3.81(\mathrm{~s}, 3 \mathrm{H})$; ${ }^{13} \mathrm{C}$ NMR (101 MHz, $\mathrm{CDCl}_{3}$ ) $\delta$ 167.6, 142.4, 134.9, 129.7 (2C), 129.2, 128.8 (2C), 128.0, 52.4, 36.7; HRMS (ESI) calculated for $\mathrm{C}_{22} \mathrm{H}_{22} \mathrm{O}_{4} \mathrm{~S}_{2} \mathrm{Na}[\mathrm{M}+\mathrm{Na}]^{+}: m / z$ 437.0857, found 437.0857.

\section{Allyl disulfane 6a}

Prepared according to GP2 using $3 \mathbf{a b}(289.6 \mathrm{mg}, 0.8 \mathrm{mmol})$ with $\mathrm{Cs}_{2} \mathrm{CO}_{3}(260.6 \mathrm{mg}, 0.8 \mathrm{mmol})$ to afford $6 \mathrm{a}(116.2 \mathrm{mg}, 44 \%)$ using $30 \%$ ethyl acetate/petether as a colorless solid. Mp: $163-165^{\circ} \mathrm{C}$. ${ }^{1} \mathrm{H}$ NMR $\left(400 \mathrm{MHz}, \mathrm{CDCl}_{3}\right) \delta 7.93(\mathrm{~s}, 1 \mathrm{H}), 7.71(\mathrm{~d}, J=8.3 \mathrm{~Hz}, 2 \mathrm{H})$, 7.50-7.43 (m, 2H), 7.40-7.33 (m, 3H), 7.27 (d, $J=8.0 \mathrm{~Hz}, 2 \mathrm{H})$, $4.47(\mathrm{~s}, 2 \mathrm{H}), 3.61(\mathrm{~s}, 3 \mathrm{H}), 2.42(\mathrm{~s}, 3 \mathrm{H}) ;{ }^{13} \mathrm{C} \mathrm{NMR}\left(101 \mathrm{MHz}, \mathrm{CDCl}_{3}\right)$ $\delta$ 167.1, 146.3, 145.5, 144.9, 136.5, 133.8, 129.8 (2C), 129.3 (2C), 128.8 (2C), 128.7 (2C), 121.2, 55.3, 52.5, 21.7; HRMS (ESI) calculated for $\mathrm{C}_{18} \mathrm{H}_{18} \mathrm{O}_{4} \mathrm{~S}_{2} \mathrm{Na}[\mathrm{M}+\mathrm{Na}]^{+}: \mathrm{m} / z$ 353.0823, found 353.0829.

\section{Diallyl disulfane $5 b$}

Prepared according to GP2 using 3db (300.6 mg, $0.8 \mathrm{mmol}$ ) with $\mathrm{Cs}_{2} \mathrm{CO}_{3}(260.6 \mathrm{mg}, 0.8 \mathrm{mmol})$ to afford $5 \mathbf{b}(80.3 \mathrm{mg}, 22.7 \% \times 2=$ $45 \%$ ) using $20 \%$ ethyl acetate/petether as a pale-yellow solid. Mp: $197-199^{\circ} \mathrm{C} ;{ }^{1} \mathrm{H}$ NMR $\left(400 \mathrm{MHz}, \mathrm{CDCl}_{3}\right) \delta 7.87-7.74(\mathrm{~m}, 1 \mathrm{H})$, $7.45(\mathrm{~d}, J=8.1 \mathrm{~Hz}, 2 \mathrm{H}), 7.19(\mathrm{~d}, J=8.0 \mathrm{~Hz}, 2 \mathrm{H}), 3.97(\mathrm{~s}, 2 \mathrm{H}), 3.81$ (s, 3H), 2.37 (s, 3H); ${ }^{13} \mathrm{C}$ NMR (101 MHz, $\left.\mathrm{CDCl}_{3}\right) \delta 167.7,142.5$, 139.6, 131.9, 129.9 (2C), 129.5 (2C), 126.9, 52.3, 37.1, 21.5; HRMS (ESI) calculated for $\mathrm{C}_{24} \mathrm{H}_{26} \mathrm{O}_{4} \mathrm{~S}_{2} \mathrm{Na}[\mathrm{M}+\mathrm{Na}]^{+}: \mathrm{m} / \mathrm{z}$ 465.1170, found 465.1172.

\section{Allyl disulfane 6b}

Prepared according to GP2 using $3 \mathbf{d b}$ (300.6 $\mathrm{mg}, 0.8 \mathrm{mmol}$ ) with $\mathrm{Cs}_{2} \mathrm{CO}_{3}(260.6 \mathrm{mg}, 0.8 \mathrm{mmol})$ to afford $6 \mathbf{b}(112.7 \mathrm{mg}, 41 \%)$ using $30 \%$ ethyl acetate/petether as a pale-yellow solid. Mp: 212- 
$214{ }^{\circ} \mathrm{C} ;{ }^{1} \mathrm{H}$ NMR $\left(400 \mathrm{MHz}, \mathrm{CDCl}_{3}\right) \delta 7.90(\mathrm{~s}, 1 \mathrm{H}), 7.72(\mathrm{~d}, J=$ $8.2 \mathrm{~Hz}, 2 \mathrm{H}), 7.41(\mathrm{~d}, J=8.0 \mathrm{~Hz}, 2 \mathrm{H}), 7.27$ (d, $J=8.0 \mathrm{~Hz}, 2 \mathrm{H}), 7.18$ $(\mathrm{d}, J=8.0 \mathrm{~Hz}, 2 \mathrm{H}), 4.48(\mathrm{~s}, 2 \mathrm{H}), 3.58(\mathrm{~s}, 3 \mathrm{H}), 2.41(\mathrm{~s}, 3 \mathrm{H}), 2.37(\mathrm{~s}$, $3 \mathrm{H}) ;{ }^{13} \mathrm{C}$ NMR $\left(101 \mathrm{MHz}, \mathrm{CDCl}_{3}\right) \delta 167.2,146.4,144.8,140.2$, 136.5, 130.9, 129.6 (2C), 129.50 (2C), 129.48 (2C), 128.6 (2C), 120.0, 55.3, 52.3, 21.7, 21.5; HRMS (ESI) calculated for $\mathrm{C}_{19} \mathrm{H}_{20} \mathrm{O}_{2} \mathrm{~S}_{2} \mathrm{Na}[\mathrm{M}+\mathrm{Na}]^{+}: \mathrm{m} / z$ 367.06980; found 367.06980.

\section{Diallyl disulfane 5c}

Prepared according to GP2 using $3 \mathrm{eb}(323.2 \mathrm{mg}, 0.8 \mathrm{mmol})$ with $\mathrm{Cs}_{2} \mathrm{CO}_{3}$ (260.6 mg, $\left.0.8 \mathrm{mmol}\right)$ to afford 5c $(79.7 \mathrm{mg}, 20 \% \times 2=$ $40 \%$ ) using $20 \%$ ethyl acetate/petether as a pale-yellow solid. Mp: $178-180{ }^{\circ} \mathrm{C} ;{ }^{1} \mathrm{H}$ NMR (400 $\left.\mathrm{MHz}, \mathrm{CDCl}_{3}\right) \delta 7.80(\mathrm{~s}, 1 \mathrm{H}), 7.50$ $(\mathrm{d}, J=8.1 \mathrm{~Hz}, 2 \mathrm{H}), 7.25(\mathrm{~d}, J=8.2 \mathrm{~Hz}, 2 \mathrm{H}), 3.96(\mathrm{~s}, 2 \mathrm{H}), 3.81(\mathrm{~s}$, $3 \mathrm{H}), 2.91$ (sept, $J=6.9 \mathrm{~Hz}, 1 \mathrm{H}), 1.25$ (d, $J=6.9 \mathrm{~Hz}, 6 \mathrm{H}) ;{ }^{13} \mathrm{C} \mathrm{NMR}$ $\left(101 \mathrm{MHz}, \mathrm{CDCl}_{3}\right) \delta 167.8,150.5,142.6,132.3,130.1$ (2C), 126.9 (2C), 126.87, 52.4, 37.1, 34.1, 23.9 (2C); HRMS (ESI) calculated for $\mathrm{C}_{28} \mathrm{H}_{35} \mathrm{O}_{4} \mathrm{~S}_{2}[\mathrm{M}+\mathrm{H}]^{+}: m / z$ 499.1957, found 499.1939 .

\section{Allyl disulfane $6 \mathrm{c}$}

Prepared according to GP2 using $3 \mathrm{eb}(323.2 \mathrm{mg}, 0.8 \mathrm{mmol})$ with $\mathrm{Cs}_{2} \mathrm{CO}_{3}(260.6 \mathrm{mg}, 0.8 \mathrm{mmol})$ to afford $6 \mathrm{c}(107.0 \mathrm{mg}, 36 \%)$ using $30 \%$ ethyl acetate/petether as a colorless solid. Mp: $216-218{ }^{\circ} \mathrm{C}$; ${ }^{1} \mathrm{H} \mathrm{NMR}\left(400 \mathrm{MHz}, \mathrm{CDCl}_{3}\right) \delta 7.94(\mathrm{~s}, 1 \mathrm{H}), 7.75(\mathrm{~d}, J=8.3 \mathrm{~Hz}, 2 \mathrm{H})$, $7.47(\mathrm{~d}, J=8.1 \mathrm{~Hz}, 2 \mathrm{H}), 7.29(\mathrm{dd}, J=8.7,2.3 \mathrm{~Hz}, 4 \mathrm{H}), 4.54(\mathrm{~s}$, $2 \mathrm{H}), 3.63(\mathrm{~s}, 3 \mathrm{H}), 3.04-2.88(\mathrm{~m}, 1 \mathrm{H}), 2.46(\mathrm{~s}, 3 \mathrm{H}), 1.30(\mathrm{~d}, J=$ $6.9 \mathrm{~Hz}, 6 \mathrm{H}) ;{ }^{13} \mathrm{C}$ NMR (101 MHz, $\mathrm{CDCl}_{3}$ ) $\delta$ 167.2, 151.1, 146.4, 144.7, 136.5, 131.3, 129.67 (2C), 129.66 (2C), 128.7 (2C), 126.9 (2C), 120.1, 55.4, 52.4, 34.1, 23.9 (2C), 21.7; HRMS (ESI) calculated for $\mathrm{C}_{21} \mathrm{H}_{24} \mathrm{O}_{2} \mathrm{SNa}[\mathrm{M}+\mathrm{Na}]^{+}: m / z$ 395.1293, found 395.1293.

\section{Diallyl disulfane 5 a}

Prepared according to GP2 using 3aa $(278.4 \mathrm{mg}, 0.8 \mathrm{mmol})$ with $\mathrm{Cs}_{2} \mathrm{CO}_{3}(260.6 \mathrm{mg}, 0.8 \mathrm{mmol})$ to afford 5a $(63.2 \mathrm{mg}, 19 \% \times 2=$ $38 \%$ ) using $20 \%$ ethyl acetate/petether as a colorless solid.

\section{Allyl disulfane 6d}

Prepared according to GP2 using 3 aa $(278.4 \mathrm{mg}, 0.8 \mathrm{mmol})$ with $\mathrm{Cs}_{2} \mathrm{CO}_{3}$ (260.6 mg, $0.8 \mathrm{mmol}$ ) to afford $6 \mathrm{~d}(83.5 \mathrm{mg}, 33 \%)$ using $30 \%$ ethyl acetate/petether as a pale-yellow viscous liquid. ${ }^{1} \mathrm{H}$ NMR (400 MHz, $\left.\mathrm{CDCl}_{3}\right) \delta 7.94(\mathrm{~s}, 1 \mathrm{H}), 7.84(\mathrm{dd}, J=8.3,1.2 \mathrm{~Hz}$, $2 \mathrm{H}), 7.60(\mathrm{t}, J=7.5 \mathrm{~Hz}, 1 \mathrm{H}), 7.51-7.45(\mathrm{~m}, 4 \mathrm{H}), 7.40-7.34(\mathrm{~m}$, $3 \mathrm{H}), 4.49(\mathrm{~s}, 2 \mathrm{H}), 3.58(\mathrm{~s}, 3 \mathrm{H}) ;{ }^{13} \mathrm{C} \mathrm{NMR}\left(101 \mathrm{MHz}, \mathrm{CDCl}_{3}\right)$ $\delta 166.9,146.4,139.4,133.8,133.7,129.8,129.2$ (2C), 129.1 (2C), 128.8 (2C), 128.5 (2C), 121.0, 55.2, 52.4; HRMS (ESI) calculated for $\mathrm{C}_{17} \mathrm{H}_{16} \mathrm{O}_{2} \mathrm{~S}_{2} \mathrm{Na}[\mathrm{M}+\mathrm{Na}]^{+}: m / z$ 339.0667, found 339.0665.

\section{Diallyl disulfane $5 \mathrm{~d}$}

Prepared according to GP2 using 3 ba $(341.6 \mathrm{mg}, 0.8 \mathrm{mmol})$ with $\mathrm{Cs}_{2} \mathrm{CO}_{3}(260.6 \mathrm{mg}, 0.8 \mathrm{mmol})$ to afford $5 \mathrm{~d}(98.6 \mathrm{mg}, 21.5 \% \times 2=$ $43 \%$ ) using $20 \%$ ethyl acetate/petether as a pale-yellow gum. ${ }^{1} \mathrm{H}$ NMR (400 MHz, $\left.\mathrm{CDCl}_{3}\right) \delta 7.72(\mathrm{~s}, 1 \mathrm{H}), 7.52(\mathrm{~d}, J=8.5 \mathrm{~Hz}, 2 \mathrm{H})$, $7.37(\mathrm{~d}, J=8.3 \mathrm{~Hz}, 2 \mathrm{H}), 3.84(\mathrm{~s}, 2 \mathrm{H}), 3.81(\mathrm{~s}, 3 \mathrm{H}) ;{ }^{13} \mathrm{C} \mathrm{NMR}(101$ $\mathrm{MHz} \mathrm{CDCl}_{3}$ ) $\delta 167.3,141.0,133.7,132.0$ (2C), 131.2 (2C), 128.6,
123.7, 52.5, 36.6; HRMS (ESI) calculated for $\mathrm{C}_{22} \mathrm{H}_{21} \mathrm{O}_{4} \mathrm{~S}_{2} \mathrm{Br}_{2}[\mathrm{M}+$ $\mathrm{H}]^{+}: m / z 570.9248$, found 570.9259 .

\section{Allyl disulfane 6e}

Prepared according to GP2 using $3 \mathbf{b a}(341.6 \mathrm{mg}, 0.8 \mathrm{mmol})$ with $\mathrm{Cs}_{2} \mathrm{CO}_{3}(260.6 \mathrm{mg}, 0.8 \mathrm{mmol})$ to afford $6 \mathrm{e}(126.3 \mathrm{mg}, 40 \%)$ using $30 \%$ ethyl acetate/petether as a pale-yellow gum. ${ }^{1} \mathrm{H}$ NMR (400 $\left.\mathrm{MHz}, \mathrm{CDCl}_{3}\right) \delta 7.87(\mathrm{~s}, 1 \mathrm{H}), 7.85(\mathrm{dd}, J=8.4,1.2 \mathrm{~Hz}, 2 \mathrm{H}), 7.66-$ $7.60(\mathrm{~m}, 1 \mathrm{H}), 7.54-7.48(\mathrm{~m}, 4 \mathrm{H}), 7.39$ (d, $J=8.3 \mathrm{~Hz}, 2 \mathrm{H}), 4.43(\mathrm{~s}$, 2H), 3.57 (s, 3H); ${ }^{13} \mathrm{C}$ NMR (101 MHz, $\left.\mathrm{CDCl}_{3}\right) \delta$ 166.7, 145.2, 139.4, 134.0, 132.6, 132.2 (2C), 130.8 (2C), 129.2 (2C), 128.6 (2C), 124.4, 121.6, 55.2, 52.6; HRMS (ESI) calculated for $\mathrm{C}_{17} \mathrm{H}_{15} \mathrm{O}_{4} \mathrm{~S}_{2^{-}}$ $\mathrm{BrNa}[\mathrm{M}+\mathrm{Na}]^{+}: m / z$ 416.9772, found 416.9776 .

\section{Diallyl disulfane 5e}

Prepared according to GP2 using $3 \mathbf{h b}(341.6 \mathrm{mg}, 0.8 \mathrm{mmol})$ with $\mathrm{Cs}_{2} \mathrm{CO}_{3}(260.6 \mathrm{mg}, 0.8 \mathrm{mmol})$ to afford $5 \mathrm{e}(89.2 \mathrm{mg}, 19.5 \% \times 2=$ $39 \%$ ) using $20 \%$ ethyl acetate/petether as a pale-yellow viscous liquid. ${ }^{1} \mathrm{H}$ NMR $\left(400 \mathrm{MHz}, \mathrm{CDCl}_{3}\right) \delta 7.78(\mathrm{~s}, 1 \mathrm{H}), 7.59(\mathrm{dd}, J=$ 8.0, $1.1 \mathrm{~Hz}, 1 \mathrm{H}), 7.45(\mathrm{dd}, J=7.6,1.3 \mathrm{~Hz}, 1 \mathrm{H}), 7.32(\mathrm{td}, J=7.5$, $0.8 \mathrm{~Hz}, 1 \mathrm{H}), 7.20(\mathrm{td}, J=7.5,1.3 \mathrm{~Hz}, 1 \mathrm{H}), 3.82(\mathrm{~s}, 3 \mathrm{H}), 3.57$ (s, $2 \mathrm{H}) ;{ }^{13} \mathrm{C}$ NMR $\left(101 \mathrm{MHz}, \mathrm{CDCl}_{3}\right) \delta 167.0,141.2,135.4,133.0$, 130.6, 130.2, 129.8, 127.5, 124.5, 52.5, 35.9; HRMS (ESI) calculated for $\mathrm{C}_{22} \mathrm{H}_{20} \mathrm{O}_{4} \mathrm{~S}_{2} \mathrm{Br}_{2} \mathrm{Na}[\mathrm{M}+\mathrm{Na}]^{+}: m / z$ 592.9063, found 592.9055 .

\section{Allyl disulfane $6 f$}

Prepared according to GP2 using $\mathbf{3 h b}(341.6 \mathrm{mg}, 0.8 \mathrm{mmol})$ with $\mathrm{Cs}_{2} \mathrm{CO}_{3}$ (260.6 mg, $\left.0.8 \mathrm{mmol}\right)$ to afford $\mathbf{6 f}(112.1 \mathrm{mg}, 34 \%)$ using $30 \%$ ethyl acetate/petether as a pale-yellow viscous liquid. ${ }^{1} \mathrm{H}$ NMR (400 MHz, $\left.\mathrm{CDCl}_{3}\right) \delta 7.94(\mathrm{~s}, 1 \mathrm{H}), 7.70(\mathrm{~d}, J=8.3 \mathrm{~Hz}, 2 \mathrm{H})$, $7.62(\mathrm{dd}, J=7.7,1.1 \mathrm{~Hz}, 1 \mathrm{H}), 7.56(\mathrm{dd}, J=8.0,1.1 \mathrm{~Hz}, 1 \mathrm{H}), 7.35$ $(\mathrm{td}, J=7.6,1.6 \mathrm{~Hz}, 1 \mathrm{H}), 7.28(\mathrm{~d}, J=8.0 \mathrm{~Hz}, 2 \mathrm{H}), 7.22(\mathrm{td}, J=7.6$, $1.2 \mathrm{~Hz}, 1 \mathrm{H}), 4.35$ (s, 2H), $3.64(\mathrm{~s}, 3 \mathrm{H}), 2.43$ (s, 3H); ${ }^{13} \mathrm{C}$ NMR (101 $\left.\mathrm{MHz}, \mathrm{CDCl}_{3}\right) \delta 166.5,145.1,144.8,136.4,134.2,133.0,130.7$, 130.2, 129.8 (2C), 128.5 (2C), 127.7, 124.2, 123.0, 55.0, 52.6, 21.7; HRMS (ESI) calculated for $\mathrm{C}_{18} \mathrm{H}_{17} \mathrm{O}_{2} \mathrm{~S}_{2} \mathrm{BrNa}[\mathrm{M}+\mathrm{Na}]^{+}: \mathrm{m} / z$ 430.9751 , found 430.9926 .

\section{Diallyl disulfane $5 f$}

Prepared according to GP2 using 30 b $(353.0 \mathrm{mg}, 0.8 \mathrm{mmol})$ with $\mathrm{Cs}_{2} \mathrm{CO}_{3}(260.6 \mathrm{mg}, 0.8 \mathrm{mmol})$ to afford $\mathbf{5 f}(108.4 \mathrm{mg}, 23.0 \% \times 2$ $=46 \%$ ) using $20 \%$ ethyl acetate/petether as a pale-yellow viscous liquid. ${ }^{1} \mathrm{H}$ NMR $\left(400 \mathrm{MHz}, \mathrm{CDCl}_{3}\right) \delta 7.77(\mathrm{~s}, 1 \mathrm{H}), 7.58$ $(\mathrm{d}, J=8.0 \mathrm{~Hz}, 1 \mathrm{H}), 7.46(\mathrm{~d}, J=7.6 \mathrm{~Hz}, 1 \mathrm{H}), 7.31(\mathrm{t}, J=7.6 \mathrm{~Hz}$, $1 \mathrm{H}), 7.19$ (t, $J=7.7 \mathrm{~Hz}, 1 \mathrm{H}), 4.28(\mathrm{q}, J=7.1 \mathrm{~Hz}, 2 \mathrm{H}), 3.59(\mathrm{~s}, 2 \mathrm{H})$, $1.33(\mathrm{t}, J=7.1 \mathrm{~Hz}, 3 \mathrm{H}) ;{ }^{13} \mathrm{C} \mathrm{NMR}\left(101 \mathrm{MHz}, \mathrm{CDCl}_{3}\right) \delta 166.5$, 140.9, 135.4, 132.9, 130.6, 130.2, 130.1, 127.4, 124.5, 61.5, 36.1, 14.4; HRMS (ESI) calculated for $\mathrm{C}_{24} \mathrm{H}_{24} \mathrm{O}_{4} \mathrm{~S}_{2} \mathrm{Br}_{2} \mathrm{Na}[\mathrm{M}+\mathrm{Na}]^{+}: \mathrm{m} / z$ 620.9380 , found 620.9373 .

\section{Allyl disulfane $6 \mathrm{~g}$}

Prepared according to GP2 using $30 b(353.0 \mathrm{mg}, 0.8 \mathrm{mmol})$ with $\mathrm{Cs}_{2} \mathrm{CO}_{3}$ (260.6 mg, $0.8 \mathrm{mmol}$ ) to afford $6 \mathrm{~g}(101.3 \mathrm{mg}, 30 \%)$ using $30 \%$ ethyl acetate/petether as a pale-yellow viscous liquid. ${ }^{1} \mathrm{H}$ 
NMR (400 MHz, $\left.\mathrm{CDCl}_{3}\right) \delta 7.94(\mathrm{~s}, 1 \mathrm{H}), 7.71(\mathrm{~d}, J=8.3 \mathrm{~Hz}, 2 \mathrm{H})$, $7.62(\mathrm{dd}, J=7.7,1.1 \mathrm{~Hz}, 1 \mathrm{H}), 7.56(\mathrm{dd}, J=8.0,1.1 \mathrm{~Hz}, 1 \mathrm{H}), 7.35$ $(\mathrm{td}, J=7.5,0.9 \mathrm{~Hz}, 1 \mathrm{H}), 7.28(\mathrm{~d}, J=8.3 \mathrm{~Hz}, 2 \mathrm{H}), 7.22(\mathrm{td}, J=7.6$, $1.3 \mathrm{~Hz}, 1 \mathrm{H}), 4.35(\mathrm{~s}, 2 \mathrm{H}), 4.10(\mathrm{q}, J=7.1 \mathrm{~Hz}, 2 \mathrm{H}), 2.43(\mathrm{~s}, 3 \mathrm{H})$, $1.26(\mathrm{t}, J=7.1 \mathrm{~Hz}, 3 \mathrm{H}) ;{ }^{13} \mathrm{C}$ NMR $\left(101 \mathrm{MHz}, \mathrm{CDCl}_{3}\right) \delta 166.0$, 144.78, 144.76, 136.5, 134.3, 132.9, 130.7, 130.2, 129.8 (2C), 128.5 (2C), 127.7, 124.2, 123.3, 61.8, 55.0, 21.7, 14.1; HRMS (ESI) calculated for $\mathrm{C}_{19} \mathrm{H}_{19} \mathrm{O}_{2} \mathrm{~S}_{2} \mathrm{BrNa}[\mathrm{M}+\mathrm{Na}]^{+}: m / z$ 445.0088, found 445.0082.

\section{Diallyl disulfane $5 \mathrm{~g}$}

Prepared according to GP2 using $3 \mathrm{gb}$ (313.6 $\mathrm{mg}, 0.8 \mathrm{mmol}$ ) with $\mathrm{Cs}_{2} \mathrm{CO}_{3}(260.6 \mathrm{mg}, 0.8 \mathrm{mmol})$ to afford $5 \mathrm{~g}(60.6 \mathrm{mg}, 16 \% \times 2=$ $32 \%)$ as a pale-yellow solid. Mp: $192-194{ }^{\circ} \mathrm{C} ;{ }^{1} \mathrm{H}$ NMR $(400 \mathrm{MHz}$, $\left.\mathrm{CDCl}_{3}\right) \delta 7.74(\mathrm{~s}, 1 \mathrm{H}), 7.25-7.19(\mathrm{~m}, 1 \mathrm{H}), 7.03(\mathrm{~d}, J=2.2 \mathrm{~Hz}, 2 \mathrm{H})$, 6.85 (dd, $J=8.1,1.9 \mathrm{~Hz}, 1 \mathrm{H}), 3.87(\mathrm{~s}, 2 \mathrm{H}), 3.76(\mathrm{~s}, 3 \mathrm{H}), 3.75$ (s, $3 \mathrm{H}) ;{ }^{13} \mathrm{C}$ NMR $\left(101 \mathrm{MHz}, \mathrm{CDCl}_{3}\right) \delta$ 167.6, 159.8, 142.5, 136.1, 129.8, 128.1, 122.1, 115.4, 114.6, 55.5, 52.4, 37.0; HRMS (ESI) calculated for $\mathrm{C}_{24} \mathrm{H}_{26} \mathrm{O}_{6} \mathrm{~S}_{2} \mathrm{Na}[\mathrm{M}+\mathrm{Na}]^{+}: m / z$ 497.1068, found 497.1069.

\section{Allyl disulfane $6 \mathrm{~h}$}

Prepared according to GP2 using $3 \mathrm{gb}$ (313.6 $\mathrm{mg}, 0.8 \mathrm{mmol}$ ) with $\mathrm{Cs}_{2} \mathrm{CO}_{3}$ (260.6 mg, $\left.0.8 \mathrm{mmol}\right)$ to afford $6 \mathrm{~h}(74.8 \mathrm{mg}, 26 \%)$ using $30 \%$ ethyl acetate/petether as a pale-yellow gum. ${ }^{1} \mathrm{H}$ NMR (400 $\left.\mathrm{MHz}, \mathrm{CDCl}_{3}\right) \delta 7.91(\mathrm{~s}, 1 \mathrm{H}), 7.72(\mathrm{~d}, J=8.2 \mathrm{~Hz}, 2 \mathrm{H}), 7.32-7.24$ $(\mathrm{m}, 3 \mathrm{H}), 7.11(\mathrm{~s}, 1 \mathrm{H}), 7.01(\mathrm{~d}, J=7.6 \mathrm{~Hz}, 1 \mathrm{H}), 6.92(\mathrm{~d}, J=8.0 \mathrm{~Hz}$, $1 \mathrm{H}), 4.48$ (s, 2H), 3.85 (s, 3H), $3.61(\mathrm{~s}, 3 \mathrm{H}), 2.43(\mathrm{~s}, 3 \mathrm{H}) ;{ }^{13} \mathrm{C}$ NMR $\left(101 \mathrm{MHz}, \mathrm{CDCl}_{3}\right) \delta 167.0,159.9,146.3,144.9,136.5,135.1$, 129.84, 129.76 (2C), 128.7 (2C), 121.7, 121.4, 116.0, 114.1, 55.6, $55.4,52.5,21.7$; HRMS (ESI) calculated for $\mathrm{C}_{19} \mathrm{H}_{20} \mathrm{O}_{5} \mathrm{SNa}[\mathrm{M}+$ $\mathrm{Na}]^{+}: m / z$ 383.0929, found 383.0937 .

\section{Diallyl disulfane $5 \mathrm{~h}$}

Prepared according to GP2 using $3 \mathbf{j b}(330.0 \mathrm{mg}, 0.8 \mathrm{mmol})$ with $\mathrm{Cs}_{2} \mathrm{CO}_{3}(260.6 \mathrm{mg}, 0.8 \mathrm{mmol})$ to afford $\mathbf{5 h}(89.3 \mathrm{mg}, 21.7 \% \times 2$ $=43 \%$ ) using $20 \%$ ethyl acetate/petether as a yellow solid. Mp: 187-198 ${ }^{\circ} \mathrm{C} ;{ }^{1} \mathrm{H}$ NMR (400 MHz, $\left.\mathrm{CDCl}_{3}\right) \delta 8.02(\mathrm{~s}, 1 \mathrm{H}), 7.95(\mathrm{~s}$, $1 \mathrm{H}), 7.79(\mathrm{dd}, J=8.7,5.3 \mathrm{~Hz}, 3 \mathrm{H}), 7.57(\mathrm{dd}, J=8.5,1.5 \mathrm{~Hz}, 1 \mathrm{H})$, 7.49 (ddd $J=8.0,6.7,1.4 \mathrm{~Hz}, 2 \mathrm{H}), 4.03(\mathrm{~s}, 2 \mathrm{H}), 3.81(\mathrm{~s}, 3 \mathrm{H}) ;{ }^{13} \mathrm{C}$ NMR (101 MHz, $\left.\mathrm{CDCl}_{3}\right) \delta 167.6,142.4,133.4,133.2,132.3,129.8$, 128.7, 128.4 128.1, 127.8, 127.2, 126.8, 126.7, 52.4, 37.2; HRMS (ESI) calculated for $\mathrm{C}_{30} \mathrm{H}_{26} \mathrm{O}_{4} \mathrm{~S}_{2} \mathrm{Na}[\mathrm{M}+\mathrm{Na}]^{+}: \mathrm{m} / z$ 537.1165, found 537.1170.

\section{Allyl disulfane 6i}

Prepared according to GP2 using $3 \mathbf{j b}(330.0 \mathrm{mg}, 0.8 \mathrm{mmol})$ with $\mathrm{Cs}_{2} \mathrm{CO}_{3}$ (260.6 mg, $0.8 \mathrm{mmol}$ ) to afford $\mathbf{6 i}(99.6 \mathrm{mg}, 33 \%)$ using $30 \%$ ethyl acetate/petether as yellow solid. Mp: $195-197{ }^{\circ} \mathrm{C} ;{ }^{1} \mathrm{H}$ NMR (400 MHz, $\left.\mathrm{CDCl}_{3}\right) \delta 8.07(\mathrm{~s}, 1 \mathrm{H}), 7.97(\mathrm{~s}, 1 \mathrm{H}), 7.88-7.78(\mathrm{~m}$, $3 \mathrm{H}), 7.70(\mathrm{~d}, J=8.3 \mathrm{~Hz}, 2 \mathrm{H}), 7.55-7.47(\mathrm{~m}, 3 \mathrm{H}), 7.18(\mathrm{~d}, J=$ $8.0 \mathrm{~Hz}, 2 \mathrm{H}), 4.57(\mathrm{~s}, 2 \mathrm{H}), 3.67(\mathrm{~s}, 3 \mathrm{H}), 2.33(\mathrm{~s}, 3 \mathrm{H}) ;{ }^{13} \mathrm{C}$ NMR $(101$ $\left.\mathrm{MHz}, \mathrm{CDCl}_{3}\right) \delta 167.2,146.2,144.9,136.5,133.6,133.2,131.3$, 129.7 (2C), 129.6, 128.8, 128.7 (2C), 128.5, 127.8, 127.5, 126.8,
126.1, 121.4, 55.4, 52.6, 21.7; HRMS (ESI) calculated for $\mathrm{C}_{22} \mathrm{H}_{20} \mathrm{O}_{2} \mathrm{~S}_{2} \mathrm{Na}[\mathrm{M}+\mathrm{Na}]^{+}: m / z$ 403.0980, found 403.0983.

\section{Diallyl disulfane 5a}

Prepared according to GP2 using 3ad (318.8 $\mathrm{mg}, 0.8 \mathrm{mmol})$ with $\mathrm{Cs}_{2} \mathrm{CO}_{3}(260.6 \mathrm{mg}, 0.8 \mathrm{mmol})$ to afford $5 \mathrm{a}(52.6 \mathrm{mg}, 15.8 \% \times 2=$ $32 \%$ ) using $20 \%$ ethyl acetate/petether as a colorless solid.

\section{Allyl disulfane $6 \mathbf{j}$}

Prepared according to GP2 using 3 ad $(318.8 \mathrm{mg}, 0.8 \mathrm{mmol})$ with $\mathrm{Cs}_{2} \mathrm{CO}_{3}(260.6 \mathrm{mg}, 0.8 \mathrm{mmol})$ to afford $6 \mathbf{j}(110.6 \mathrm{mg}, 38 \%)$ using $30 \%$ ethyl acetate/petether as a pale-yellow solid. Mp: 227$229{ }^{\circ} \mathrm{C} ;{ }^{1} \mathrm{H}$ NMR $\left(400 \mathrm{MHz}, \mathrm{CDCl}_{3}\right) \delta 8.38(\mathrm{~s}, 1 \mathrm{H}), 7.97-7.87(\mathrm{~m}$, $4 \mathrm{H}), 7.79$ (dd, $J=8.7,1.8 \mathrm{~Hz}, 1 \mathrm{H}), 7.69-7.57$ (m, 2H), 7.37 (dd, $J$ $=7.4,1.6 \mathrm{~Hz}, 2 \mathrm{H}), 7.27-7.22(\mathrm{~m}, 3 \mathrm{H}), 4.57(\mathrm{~s}, 2 \mathrm{H}), 3.45(\mathrm{~s}, 3 \mathrm{H})$; ${ }^{13} \mathrm{C}$ NMR $\left(101 \mathrm{MHz}, \mathrm{CDCl}_{3}\right) \delta 166.9,146.3,136.2,135.4,133.6$, 132.1, 130.5, 129.6, 129.5, 129.4, 129.3, 129.0 (2C), 128.7 (2C), 128.0, 127.7, 123.2, 121.1, 55.1, 52.4; HRMS (ESI) calculated for $\mathrm{C}_{21} \mathrm{H}_{19} \mathrm{O}_{4} \mathrm{~S}_{2}[\mathrm{M}+\mathrm{H}]^{+}: m / z$ 367.1004, found 367.1005.

\section{Diallyl disulfane $5 i$}

Prepared according to GP2 using $3 \mathbf{n b}(300.8 \mathrm{mg}, 0.8 \mathrm{mmol})$ with $\mathrm{Cs}_{2} \mathrm{CO}_{3}(260.6 \mathrm{mg}, 0.8 \mathrm{mmol})$ to afford $5 \mathbf{i}(70.7 \mathrm{mg}, 20 \% \times 2=$ $40 \%$ ) using $20 \%$ ethyl acetate/petether as a pale-yellow liquid. ${ }^{1} \mathrm{H} \mathrm{NMR}\left(400 \mathrm{MHz}, \mathrm{CDCl}_{3}\right) \delta 7.81(\mathrm{~s}, 1 \mathrm{H}), 7.50(\mathrm{~d}, J=6.9 \mathrm{~Hz}, 2 \mathrm{H})$, $7.40-7.33(\mathrm{~m}, 3 \mathrm{H}), 4.27(\mathrm{q}, J=7.1 \mathrm{~Hz}, 2 \mathrm{H}), 3.89(\mathrm{~s}, 2 \mathrm{H}), 1.32(\mathrm{t}, J$ $=7.1 \mathrm{~Hz}, 3 \mathrm{H}) ;{ }^{13} \mathrm{C} \mathrm{NMR}\left(101 \mathrm{MHz}, \mathrm{CDCl}_{3}\right) \delta 167.1,142.1,134.9$, 129.7, 129.1 (2C), 128.8, 128.3 (2C), 61.4, 36.8, 14.4; HRMS (ESI) calculated for $\mathrm{C}_{24} \mathrm{H}_{27} \mathrm{O}_{4} \mathrm{~S}_{2}[\mathrm{M}+\mathrm{H}]^{+}: \mathrm{m} / \mathrm{z}$ 443.1334, found 443.1351 .

\section{Allyl disulfane 6k}

Prepared according to GP2 using $3 \mathbf{n b}$ (300.8 $\mathrm{mg}, 0.8 \mathrm{mmol}$ ) with $\mathrm{Cs}_{2} \mathrm{CO}_{3}(260.6 \mathrm{mg}, 0.8 \mathrm{mmol})$ to afford $6 \mathbf{k}(96.5 \mathrm{mg}, 35 \%)$ using $30 \%$ ethyl acetate/petether as a pale-yellow liquid. ${ }^{1} \mathrm{H}$ NMR (400 $\left.\mathrm{MHz} \mathrm{CDCl}_{3}\right) \delta 7.91(\mathrm{~s}, 1 \mathrm{H}), 7.71(\mathrm{~d}, J=8.3 \mathrm{~Hz}, 2 \mathrm{H}), 7.50-7.43$ $(\mathrm{m}, 2 \mathrm{H}), 7.39-7.33(\mathrm{~m}, 3 \mathrm{H}), 7.26(\mathrm{~d}, J=8.0 \mathrm{~Hz}, 2 \mathrm{H}), 4.48(\mathrm{~s}, 2 \mathrm{H})$, 4.07 (q, $J=7.1 \mathrm{~Hz}, 2 \mathrm{H}), 2.41(\mathrm{~s}, 3 \mathrm{H}), 1.24(\mathrm{t}, J=7.1 \mathrm{~Hz}, 3 \mathrm{H}) ;{ }^{13} \mathrm{C}$ NMR (101 MHz, $\left.\mathrm{CDCl}_{3}\right) \delta 166.7,146.0,144.8,136.5,133.9,129.7$ (2C), 129.6, 129.3 (2C), 128.8 (2C), 128.7 (2C), 121.6, 61.7, 55.2, 21.7, 14.2; HRMS (ESI) calculated for $\mathrm{C}_{19} \mathrm{H}_{20} \mathrm{O}_{2} \mathrm{~S}_{2} \mathrm{Na}[\mathrm{M}+\mathrm{Na}]^{+}$: $\mathrm{m} / \mathrm{z} 367.0980$, found 367.0981 .

\section{Conclusions}

We have successfully developed a convenient protocol for the synthesis of allyl thiosulfonates. A range of aryl/heteroaryl/ aliphatic allyl bromides and sodium arylthiosulfonates were readily assembled to furnish allyl thiosulfonates. $\mathrm{Cs}_{2} \mathrm{CO}_{3}$ serves as an efficient reagent for the synthesis of diallyl disulfanes and allyl disulfanes in moderate to high yields under mild reaction conditions. The present methodologies are operationally simple, tolerate a broad range of functional groups and are also reliable in a gram scale reaction for the synthesis of thiosulfonates. 


\section{Conflicts of interest}

There are no conflicts to declare.

\section{Acknowledgements}

We thank SERB-ECR (File No. ECR/2015/000053), New Delhi for financial assistance. Our special thanks to OUDST-PURSE Programme for generous funding. R. J. R. thanks to UGC for faculty position under Faculty Recharge Programme. M. W. and J. J. K. thanks to UGC and DST Inspire, New Delhi, respectively for their research fellowship. We also thank OUCFRD for NMR facility.

\section{Notes and references}

1 Leading reviews: (a) C. Ni, M. Hu and J. Hu, Chem. Rev., 2015, 115, 765; (b) J. E. M. D. McReynolds, J. M. Dougherty and P. R. Hanson, Chem. Rev., 2004, 104, 2239; (c) Organosulfur Chemistry I \& II, ed. P. C. B. Page, Springer, Berlin, 1999.

2 Review articles: (a) C.-F. Lee, R. S. Basha and S. S. Badsara, Top. Curr. Chem., 2018, 376, 25; (b) K. L. Dunbar, D. H. Scharf, A. Litomska and C. Hertweck, Chem. Rev., 2017, 117, 5521; (c) X.-H. Xu, K. Matsuzaki and N. Shibata, Chem. Rev., 2015, 115, 731; (d) C.-F. Lee, Y.-C. Liu and S. S. Badsara, Chem.-Asian J., 2014, 9, 706; (e) H. Liu and X. Jiang, Chem.-Asian J., 2013, 8, 2546.

3 (a) P. K. Shyam and H.-Y. Jang, J. Org. Chem., 2017, 82, 1761; (b) K. Kanemoto, Y. Sugimura, S. Shimizu, S. Yoshida and T. Hosoya, Chem. Commun., 2017, 53, 10640; (c) W. Wang, X. Peng, F. Wei, C.-H. Tung and Z. Xu, Angew. Chem., Int. Ed., 2016, 55, 649; (d) S. Yoshida, Y. Sugimura, Y. Hazama, Y. Nishiyama, T. Yano, S. Shimizu and T. Hosoya, Chem. Commun., 2015, 51, 16613; (e) P. Mampuys, Y. Zhu, T. Vlaar, E. Ruijter, R. V. A. Orru and B. U. W. Maes, Angew. Chem., Int. Ed., 2014, 53, 12849.

4 Recent examples: (a) Y. Yu, Y. Zhou, Z. Song and G. Liang, Org. Biomol. Chem., 2018, 16, 4958; (b) F. Xiao, D. Wang, S. Yuan, H. Huang and G.-J. Deng, RSC Adv., 2018, 8, 23319; (c) Y. Siddaraju and K. R. Prabhu, Org. Biomol. Chem., 2017, 15, 5191; (d) P. Singh, R. Bai, R. Choudhary, M. C. Sharma and S. S. Badsara, RSC Adv., 2017, 7, 30594; (e) J. Sun, D. Zhang-Negrerie and Y. Du, Adv. Synth. Catal., 2016, 358, 2035; (f) S.-r. Guo, Y.-q. Yuan and J.-n. Xiang, Org. Lett., 2013, 15, 4654.

5 (a) N. S. Zefirof, N. V. Zyk, E. K. Beloglazkina and A. G. Kutateladze, Sulfur Rep., 1993, 14, 223; (b) J. P. Weidner and S. S. Block, J. Med. Chem., 1964, 7, 671.

6 Synthesis of thiosulfonates from disulfides and thiols oxidation, see: (a) P. K. Shyam, Y. K. Kim, C. Lee and H.-Y. Jang, Adv. Synth. Catal., 2016, 358, 56; (b) P. Natarajan, Tetrahedron Lett., 2015, 56, 4131; (c) T. X. T. Luu, T.-T. T. Nguyen, T. N. Le, J. Spanget-Larsen and F. Duus, J. Sulfur Chem., 2015, 36, 340; (d) M. Kirihara, S. Naito, Y. Nishimura, Y. Ishizuka, T. Iwai, H. Takeuchi, T. Ogata, H. Hanai, Y. Kinoshita, M. Kishida, K. Yamazaki, T. Noguchi and S. Yamashoji, Tetrahedron, 2014, 70, 2464.
7 Recent reports for thiosulfonates from sulfinate salts, see: $(a)$ N. Taniguchi, J. Org. Chem., 2015, 80, 1764; (b) N. Taniguchi, Eur. J. Org. Chem., 2014, 5691; (c) G. Liang, M. Liu, J. Chen, J. Ding, W. Gao and H. Wu, Chin. J. Chem., 2012, 30, 1611; (d) G. Liang, J. Chen, J. Chen, W. Li, J. Chen and H. Wu, Tetrahedron Lett., 2012, 53, 6768.

8 Recent examples using sulfonyl chlorides and hydrazines, see: (a) Z. Peng, X. Zheng, Y. Zhang, D. An and W. Dong, Green Chem., 2018, 20, 1760; (b) Q. Chen, Y. Huang, X. Wang, J. Wu and G. Yu, Org. Biomol. Chem., 2018, 16, 1713; (c) G.-Y. Zhang, S.-S. Lv, A. Shoberu and J.-P. Zou, J. Org. Chem., 2017, 82, 9801; (d) X. Zhao, T.-X. Liu and G. Zhang, Asian J. Org. Chem., 2017, 6, 677; (e) Y. Zheng, F.-L. Qing, Y. Huang and X.-H. Xu, Adv. Synth. Catal., 2016, 358, 3377.

9 (a) M. Musiejuk, J. Doroszuk and D. Witt, RSC Adv., 2018, 8, 9718; (b) M. Salvadl, B. Amgarten, S. Castillln, G. J. L. Bernardes and O. Boutureira, Org. Lett., 2015, 17, 2836; (c) D. P. Gamblin, P. Garnier, S. J. Ward, N. J. Oldham, A. J. Fairbanks and B. G. Davis, Org. Biomol. Chem., 2003, 1, 3642; (d) S. Takano, K. Hiroya and K. Ogasawara, Chem. Lett., 1983, 255; (e) A. P. Kozikowskif, A. Ames and H. Wetter, J. Organomet. Chem., 1979, 164, C33 and also see ref. $3 b$.

10 (a) E. Block, Angew. Chem., Int. Ed., 1992, 31, 1135; (b) S. Wang and H. Kohn, J. Med. Chem., 1999, 42, 788; (c) T. T. Conway, E. G. DeMaster, D. J. W. Goon, F. N. Shirota and H. T. Nagasawa, J. Med. Chem., 1999, 42, 4016; (d) C. Wright and R. D. Moore, Am. J. Med., 1990, 88, 647; (e) S. A. Caldarelli, M. Hamel, J.-F. Duckert, M. Ouattara, M. Calas, M. Maynadier, S. Wein, C. Périgaud, A. Pellet, H. J. Vial and S. Peyrottes, J. Med. Chem., 2012, 55, 4619.

11 (a) M. Song, J.-S. Kim, L. Liu, M. Husain and A. VázquezTorres, Cell Rep., 2016, 14, 2901; (b) T. Ilani, A. Alon, I. Grossman, B. Horowitz, E. Kartvelishvily, S. R. Cohen and D. Fass, Science, 2013, 341, 74; (c) J. Alegre-Cebollada, P. Kosuri, J. A. Rivas-Pardo and J. M. Fernández, Nat. Chem., 2011, 3, 882; (d) M. Góngora-Benítez, J. Tulla-Puche and F. Albericio, Chem. Rev., 2014, 114, 901.

12 (a) B. Mandal and B. Basu, RSC Adv., 2014, 4, 13854 and references herein; (b) T. M. Postma and F. Albericio, Eur. J. Org. Chem., 2014, 3519.

13 Selected reviews on $\mathrm{MBH}$ reaction, see: (a) K. Kaur and I. N. N. Namboothiri, Chimia, 2012, 66, 913; (b) D. Basavaiah, B. S. Reddy and B. S. Badsara, Chem. Rev., 2010, 110, 5447; (c) V. Declerck, J. Martinez and F. Lamaty, Chem. Rev., 2009, 109, 1; (d) V. Singh and S. Batra, Tetrahedron, 2008, 64, 4511; (e) D. Basavaiah, K. V. Rao and R. J. Reddy, Chem. Soc. Rev., 2007, 36, 1581.

14 Synthesis of sodium thiosulfonates, (a) R. Sato, T. Goto, Y. Takikawa and S. Takizawa, Synthesis, 1980, 615; (b) A. J Fischmanna and L. Spiccia, Dalton Trans., 2011, 40, 12310. 15 (a) R. J. Reddy, Md. Waheed, T. Karthik and A. Shankar, New J. Chem., 2018, 42, 980; (b) R. J. Reddy, A. Shankar, M. Waheed and J. B. Nanubolu, Tetrahedron Lett., 2018, 59, 2014. 
16 The regioselectivities of thiosulfonates based on the corresponding $\mathrm{MBH}$ allyl bromides (as a mixture of $E$ and $Z$ isomers), see: D. Basavaiah, K. R. Reddy and N. Kumaragurubaran, Nat. Protoc., 2007, 2, 2665.
17 (a) M. J. Cha, Y. S. Song, E.-G. Han and K.-J. Lee, J. Heterocycl. Chem., 2008, 45, 235; (b) M. J. Cha, Y. S. Song and K.-J. Lee, Bull. Korean Chem. Soc., 2006, 27, 1900.

18 X. Xiao, M. Feng and X. Jiang, Angew. Chem., Int. Ed., 2016, 55, 14121. 